University of Nebraska - Lincoln

DigitalCommons@University of Nebraska - Lincoln

$6-2005$

\title{
A Protocol for the Staining of Cement Lines in Adult Human Bone Using Toluidine Blue
}

Daniel L. Osborne

University of Nebraska - Lincoln, dosborne2@unl.edu

Janene Curtis

University of Alberta

Follow this and additional works at: https://digitalcommons.unl.edu/anthropologyfacpub

Part of the Anthropology Commons

Osborne, Daniel L. and Curtis, Janene, "A Protocol for the Staining of Cement Lines in Adult Human Bone Using Toluidine Blue" (2005). Anthropology Faculty Publications. 34.

https://digitalcommons.unl.edu/anthropologyfacpub/34

This Article is brought to you for free and open access by the Anthropology, Department of at DigitalCommons@University of Nebraska - Lincoln. It has been accepted for inclusion in Anthropology Faculty Publications by an authorized administrator of DigitalCommons@University of Nebraska - Lincoln. 


\title{
A Protocol for the Staining of Cement Lines in Adult Human Bone Using Toluidine Blue
}

\author{
Daniel L. Osborne ${ }^{1 *}$ and Janene Curtis ${ }^{2}$ \\ ${ }_{1}^{1}$ Department of Anthropology, Indiana University, Bloomington, IN \\ ${ }^{2}$ Department of Anthropology, University of Alberta, Edmonton, AB, Canada
}

\begin{abstract}
Increased definition of cement lines aids in the identification of secondary osteons in bone. A staining protocol taken from Burr (Anat Rec 232:180-189, 1991) and modified by Havill using toluidine blue on plastic-embedded bone specimens was applied to cranial core and femoral shaft sections but proved to be too time-consuming and inconsistent. Experimentation resulted in a different protocol for each bone type, perhaps as the result of differences in section thickness and cortical area. Staining of femoral sections proved particularly difficult, with increased etching duration and agitation proving most influential in intensity and uniformity of stain. (The J Histotechnol 28:73, 2005)
\end{abstract}

Submitted August 20, 2004; accepted with revisions April 15,2005

Key words: bone histology, cement lines, plastic embedding, stain, toluidine blue

\section{Introduction}

Several methods have been used to stain undecalcified bone tissue in an attempt to observe cement lines and osteons more clearly. Cresyl fast violet, thionin dye (1), and Villanueva's bone stain (2) have been used to successfully identify cement lines and demarcate complete and fragmentary osteons in fixed osseous tissue. Basic fuchsin stain, although useful on fresh, unfixed tissue $(3,4)$, has proven ineffective for highlighting cement lines in cranial and femoral bone from cadavers $(5,6)$.

Schenk et al. (7) developed a staining protocol using toluidine blue for observing cement lines in thin $(1-3 \mu \mathrm{m})$ sections; this was subsequently modified for thick sections of desiccated bone tissue from the femoral midshaft of macaques $(8,9)$. Schenk's toluidine blue protocol proved to be time consuming and inconsistent when applied to bone from embalmed specimens.

Toluidine blue is useful in the identification of cement lines, which turn dark blue subsequent to staining. Cement

* Address reprint requests to Daniel L. Osborne, Department of Anthropology, Indiana University, Bloomington, IN 47405. E-mail: dosborne@indiana.edu lines separate secondary osteons (10), and although the biomechanical function of cement lines continues to be debated (11), it is generally agreed that their composition is distinct from that of lamellar bone (11-13). This study examines the effectiveness of toluidine blue (Sigma-Aldrich, St. Louis, $\mathrm{MO}$ ) as a stain for cadaver bone tissue, based on earlier protocols for fresh bone and provides modification to earlier protocols to improve consistency and time-efficiency. Cranial core and femoral sections are used to investigate variation in staining success using toluidine blue.

\section{Materials and Methods}

Full-thickness cores from the frontal bone of the calvarium and complete cross-sections from the femoral midshaft were taken from embalmed cadavers and embedded in methyl methacrylate (MMA). Material was dehydrated through a graded series of ethanols (70\%, 80\% [two changes], $95 \%$ [two changes], $100 \%$ [two changes]), cleared in xylenes, and infiltrated with unpolymerized MMA. All steps were performed for $4 \mathrm{~h}$ under vacuum $(17 \mathrm{in} \mathrm{Hg}$ ) at room temperature (RT). The material was then infiltrated under vacuum at $\mathrm{RT}$ for $7+$ days in a mixture of MMA + 3\% dibutyl pthalate. Finally, the material was embedded using a mixture of MMA $+3 \%$ dibutyl pthalate $+0.25 \%$ Perkadox and left to polymerize at RT.

It is possible that fluids used in embalming could influence staining success, but the material used in this analysis was derived from several different medical science programs and therefore could not be controlled for. The material used in this analysis was derived from older adult individuals. Each block was sectioned with a diamond wire saw (Histosaw, Delaware Diamond Knives, Wilmington, $\mathrm{DE})$ at a thickness ranging from 70 to $90 \mu \mathrm{m}$ for frontal bones and $90-120 \mu \mathrm{m}$ for femoral bone. Sections were placed into tissue cassettes before processing.

Bone sections from the frontal bone and femur were stained with toluidine blue according to Schenk's toluidine blue protocol (7), with minor modifications $(8,9)$. The modifications to the original protocol included increasing the amount of time sections were submerged in formic acid $(0.1 \%)$ and toluidine blue solutions, as well as the use of EtOH (70\%) instead of methanol (20\%; Table 1). 
Table 1. Original Toluidine Blue Protocol ${ }^{\mathrm{a}}$

\begin{tabular}{ll}
\hline Solution & Immersion time \\
\hline (1) Formic acid 0.1\% & 3 min \\
(2) $\mathrm{dH}_{2} \mathrm{O}$ & Quick rinse \\
(3) $70 \%$ EtOH & $15 \mathrm{~min}$ \\
(4) $\mathrm{Toluidine}$ blue $1 \%$ solution $^{\mathrm{b}}$ & $1 \mathrm{~h}$ \\
(5) $\mathrm{dH}_{2} \mathrm{O}$ & Quick dip \\
(6) $95 \%$ EtOH & Quick dip \\
(7) $99 \%$ isopropyl & $1 \mathrm{~min}$ \\
(8) $99 \%$ isopropyl & $1 \mathrm{~min}$ \\
(9) Xylene & $3 \mathrm{~min}$ \\
(10) Xylene & $3 \mathrm{~min}$ \\
(11) Xylene & $3 \mathrm{~min}$ \\
\hline
\end{tabular}

${ }^{a}$ Burr et al (8); Havill (9).

toluidine blue solution at $1 \%$ toluidine blue, $1 \%$ sodium tetraborate hydrous.

Also, the modified protocol used a dehydration component absent from the original protocol to increase differentiation. Subsequent to differentiation, the sections were blotted dry with a paper towel and then stacked and weighted for 3 days to flatten the sections. Sections were then cleared in xylene for $30 \mathrm{~s}$, mounted with Eukitt's adhesive onto individual glass slides, and coverslipped. Because the sections were thick, the glass slide and coverslip were held in place with a weight for 2-3 days until dry.

In an attempt to identify cement lines in bone, the staining protocol as modified by Burr et al. (8) and Havill (9) was used for analysis of cranial core sections. However, use of this protocol failed to stain osseous tissue, although associated soft tissue absorbed the solution sufficiently. Given the difficulty and time investment involved in sectioning femoral shafts, individual sections were quartered for use in staining experimentation. Unlike the frontal bone sections, which were processed individually, femoral sections were processed collectively.

The formic acid solution $(0.1 \%)$ consisted of $0.1 \mathrm{~mL}$ of formic acid ( $88 \%$ stock solution) and $100 \mathrm{~mL}$ of $\mathrm{dH}_{2} \mathrm{O}$. The toluidine blue solution (1\%) consisted of $1 \mathrm{~g}$ of toluidine blue, $1 \mathrm{~g}$ of sodium borate anhydrous, and $100 \mathrm{~mL}$ of $\mathrm{dH}_{2} \mathrm{O}$. The $\mathrm{pH}$ of the toluidine blue solution was approximately 9.0. In this analysis, the hydration factor was sodium borate decahydrate. The concentrations of the formic acid and toluidine blue solutions were held constant in the inquiries below.

Assessment of the sections was qualitative in nature. The optimally stained section was dark enough to improve identification of cement lines relative to the unstained section, yet not overly dark such that the surrounding bone matrix hindered observation. In this sense distinguishing between cement lines and matrix also played a part in the assessment of quality. Finally, it was considered advantageous if the section was uniformly stained and free of excess debris resulting from the sectioning process.

\section{Experiments}

\section{Experiment A: Stain Duration}

The first experiment tested variation in staining intensity with varying time in the toluidine blue solution. To investigate this, one section was submerged in toluidine blue for
$5 \mathrm{~min}$ while another was exposed to the solution for $15 \mathrm{~min}$. For each specimen, acid etching was held at $3 \mathrm{~min}$.

\section{Experiment B: Acid Etching}

The effects of acid etching (AE) were also investigated. In this context etching refers to the decalcification of bone at the level of the embedded section. This experimental design held staining time at $15 \mathrm{~min}$ while varying the duration of etching using formic acid $(0.1 \% ; 0 \mathrm{~min}[3 \mathrm{~min}$ in $\mathrm{dH}_{2} \mathrm{O}$, $3 \mathrm{~min}, 5 \mathrm{~min}$, and $15 \mathrm{~min}$ ).

\section{Experiment C: Agitation}

A further series of experiments to assess the effects of agitation were undertaken to further optimize staining procedures. In this experiment, one section received agitation using a shaker plate $(\sim 60 \mathrm{rpm})$ while in the toluidine blue solution, whereas the other section received agitation during exposure to formic acid $(0.1 \%)$, EtOH $(70 \%)$, and toludine blue steps. For each section, etching and staining times were held at 3 and $5 \mathrm{~min}$, respectively.

\section{Experiment D: Etching and Stain Duration With Agitation}

Upon discovering the influence of agitation on staining intensity, the interactions between $\mathrm{AE}$ and staining times with agitation were examined. Both AE times (5 and 10 $\mathrm{min}$ ) and staining times (10 and $15 \mathrm{~min}$ ) were manipulated in a series of four experiments (Table 2). Given that agitation increases staining intensity, a series of tests were performed to investigate how restricting agitation to the staining component of the protocol compared to agitation at all stages. Etching time was held at $3 \mathrm{~min}$, whereas staining time and agitation were varied (Table 3). To examine the interaction between $\mathrm{AE}$ duration and agitation three experiments were performed, varying etching duration $(2,3$, and 4 min; randomly chosen) while maintaining agitation at every stage of the protocol.

\section{Experiment E: Differentiation}

Comparisons were made between the differentiated and undifferentiated frontal bone groups, choosing the appropriate staining time and method based on the best resolution

Table 2. Interaction between Etching Time and Staining Time With Agitation

\begin{tabular}{lc}
\hline Etching $(\mathrm{min})$ & Stain duration $(\mathrm{min})$ \\
\hline 5 & 5 \\
5 & 15 \\
10 & 5 \\
10 & 15 \\
\hline
\end{tabular}

Table 3. Agitation Stage and Staining Duration

\begin{aligned} & \hline Time in toluidine blue Agitation stage \\ & \hline 5 Toluidine blue only \\ & 5 Entire procedure \\ & 15 Toluidine blue only \\ & 15 Entire procedure \\ & \hline\end{aligned}


and demarcations of the structures within the bone tissue. In this context, differentiation refers to the removal of stain from the bone matrix except for the feature(s) of interest, in this case: cement lines. In the first group of sample sections, differentiation with $70 \% \mathrm{EtOH}$ to remove excess stain and decolorize the majority of the bone matrix (14) took place subsequent to staining, whereas the second group of sample sections remained undifferentiated.

\section{Experiment F: Alcohol Duration}

In an attempt to minimize overall staining time, the effects of varying alcohol duration under full agitation were investigated. Immersion in alcohol can also serve to etch the section by softening the embedding media (i.e., MMA), thereby permitting the dye better access to the tissue. To determine the optimal duration of the alcohol step, sections were stained under identical conditions with the exception of the alcohol stage (Table 4).

\section{Experiment G: Shelf Life}

The age of stain was examined as a potential influence on staining success. Previous experience suggested that toluidine blue has a limited shelf life; thus, sections of the same specimen were processed with solutions mixed 5 weeks and 2 weeks before staining.

\section{Results}

\section{Experiment A: Stain Duration}

The results suggest more variation within individual specimens than between specimens stained for 5 and 15 min, suggesting that staining duration does not influence demarcation of cement lines (Table 5; Figures 1, A and B).

\section{Experiment B: Acid Etching}

Results suggest a relationship between AE duration and staining intensity, with the darkest specimens having been etched for the longest period of time (Table 5; Figure 1, $\mathrm{C}-\mathrm{F}$ ). Unetched specimens demonstrated no staining of osseous tissue, although the associated soft tissue was stained. Variation in staining success was highly variable within individual sections independent of etching duration.

\section{Experiment C: Agitation}

Each protocol produced uniform staining, but also produced an unexpected result as the staining intensity also increased with agitation (Table 5; Figure 2, A and B). As these figures demonstrate, staining intensity is greater in the specimen that was agitated during exposure to formic acid $(0.1 \%)$, EtOH $(70 \%)$, and toludine blue.

\section{Table 4. Effects of Alcohol Duration Under Full Agitation}

\begin{tabular}{lcc}
\hline $\begin{array}{l}\text { Time in } \\
\text { toluidine blue }\end{array}$ & Acid etching & Alcohol \\
\hline 3 & 5 & 0 \\
3 & 5 & 5 \\
3 & 5 & 10 \\
3 & 5 & 15 \\
\hline
\end{tabular}

Table 5. Staining Results by Experiment

\begin{tabular}{lcl}
\hline Experiment & Figure & Result \\
\hline A & 1A & Too light \\
A & 1B & Adequate \\
B & 1C & Unstained \\
B & ID & Too light \\
B & $1 \mathrm{E}$ & Too light \\
B & 1F & Too dark \\
C & 2A & Good \\
C & 2B & Adequate \\
D & 2C & Dark \\
D & 2D & Dark \\
D & 2E & Too dark \\
D & 2F & Too dark \\
D & $3 \mathrm{~A}$ & Good \\
D & 3B & Good \\
D & $3 \mathrm{C}$ & Too light \\
D & 3D & Good \\
D & 3F & Good \\
F & $4 \mathrm{~A}$ & Unclear \\
F & $4 \mathrm{~B}$ & Unclear \\
F & $4 \mathrm{C}$ & Better \\
G & 4D & Best \\
\hline
\end{tabular}

\section{Experiment D: Etching and Stain Duration With Agitation}

These procedures resulted in uniformly stained sections that were too intensely stained for optimal definition of cement lines. Furthermore, the duration of staining did not substantially affect staining intensity, as sections stained at 5 and 15 min were of equal intensity (Table 5; Figure 2, $\mathrm{C}-\mathrm{F}$ ). However, etching time greatly influenced staining success, as increased etching removes a greater amount of calcium and MMA allowing the dye to penetrate deeper into the section, thereby increasing staining intensity.

Once again the importance of agitation was demonstrated, as sections agitated throughout the protocol were darker than sections agitated during individual components of the staining protocol (Table 5; Figures 1 [A and B] and $3[\mathrm{~A}$ and $\mathrm{B}]$ ). Variation in staining duration produced no differences. In the 5- and 15-min staining protocols, sections agitated during exposure to toluidine blue only were lighter than those agitated throughout the duration of the procedure, but both resulted in usable specimens. To increase intensity of stain it is suggested to increase etching time.

The section etched for 2 min was markedly lighter and did not define the cement lines well. Etching for $4 \mathrm{~min}$ produced the best results with better definition of the cement line, but was not noticeably different from the section etched for 3 min (Table 5; Figure 3, C-E).

\section{Experiment E: Differentiation}

Optimal results were produced by staining the sections in toluidine blue for $5 \mathrm{~min}$ followed by the differentiation step for $30 \mathrm{~s}$.

\section{Experiment F: Alcohol Duration}

Results suggest a graded effect, with increased time in alcohol producing more constant and intense staining of the 

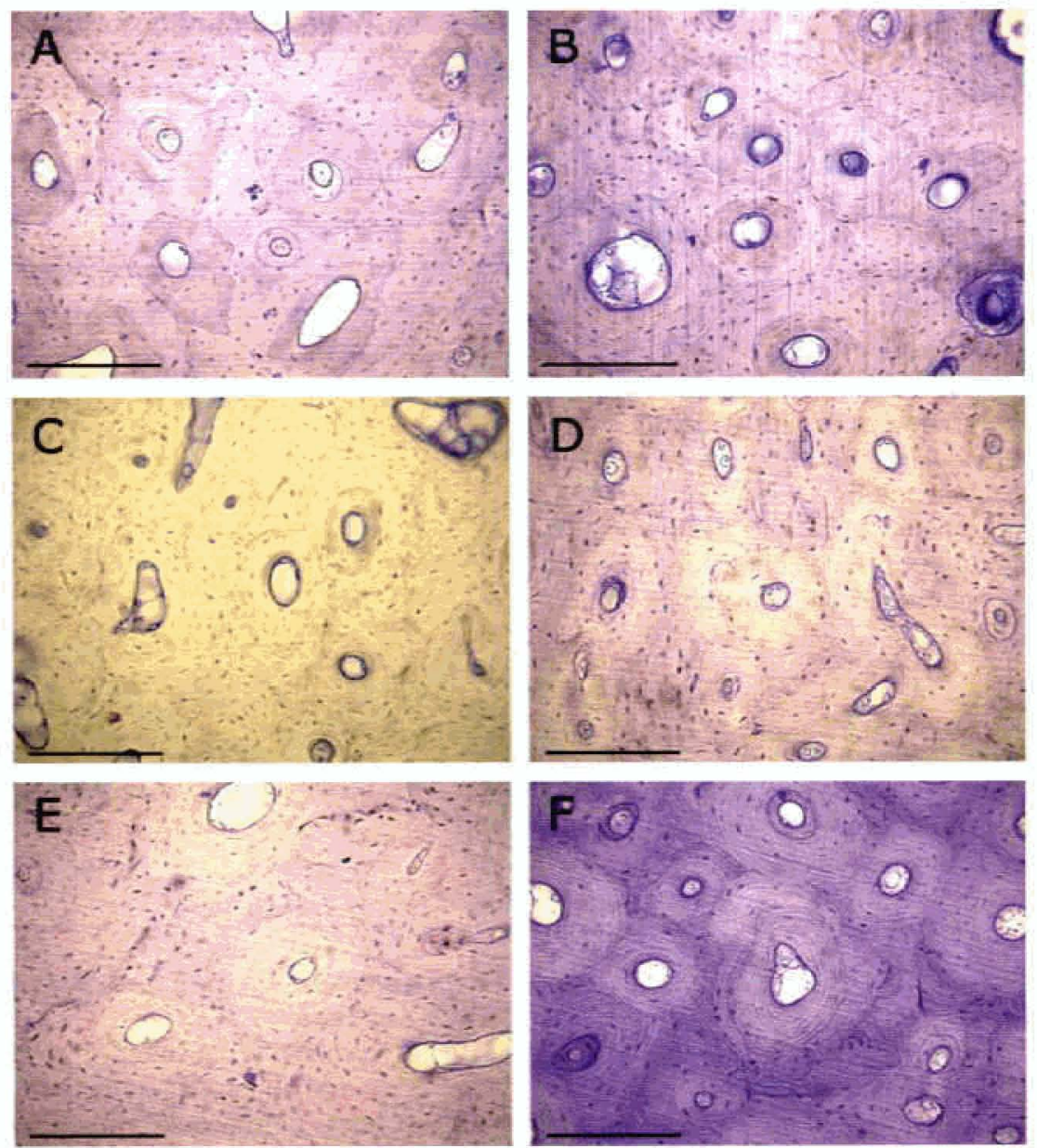

Figure 1. Photomicrographs of bone stained with toluidine blue taken with a $\times 10$ objective; scale is ecuivalent to 250 microns. A, femoral section exposed to toluidine blue for a period of $5 \mathrm{~min}$. B, femoral section exposed to toluidine blue for a period of $15 \mathrm{~min}$. C, femoral section stained without acid etching. D, femoral section etched for $3 \mathrm{~min}$. E, femoral section etched for $5 \mathrm{~min}$. F, femoral section etched for $15 \mathrm{~min}$.

cement line (Table 5; Figure 4. A-D). That is, there were no differences in staining intensity between sections, but in the differentiation of cement lines resulting from increased alcohol exposure. It is useful to note, however, that the difference in quality between sections stained at 10 and $15 \mathrm{~min}$ was negligible.

\section{Experiment G: Shelf Life}

Results suggest a substantial difference between these sections, with the older solution demonstrating a weakening of staining intensity relative to the newer solution.

\section{Discussion}

The goal of this work was to discover an optimal staining protocol for the demarcation of cement lines using a toluidine blue solution ( $\mathrm{pH} 9.0$ ). The final staining protocol for bone sections embedded in thick plastic (e.g., 80-120 $\mu \mathrm{m}$ ) is provided in Table 6. Standards for staining with and without agitation are provided, although only the time involved during the etching stage differs between these two. However, agitation results in a more uniform staining as well as a decrease in the amount of particulate matter remaining on the section once processed. The protocol resulting from this analysis produces uniform staining of cement lines in a much shorter amount of time relative to earlier protocols $(8,9)$.

Etching duration proved to play an important role in staining success, with increased etching time resulting in greater staining intensity. This is particularly true when coupled with agitation. The interaction between agitation and staining intensity is likely a product of local $\mathrm{pH}$. Whereas etching without agitation produces equilibrium, agitation permits a turning over of this interface resulting in increased surface etching.

Although etching duration greatly influenced staining success, the amount of staining time made little difference in staining intensity, with more variation within sections than between sections. This likely reflects a saturation effect with the solution staining the exposed surface. Under this scenario etching duration removes surface mineral, allowing greater access of the stain and thereby determining staining intensity. Although staining duration does not appear to affect staining intensity, age of the stain does influence the quality of the results. It is safe to assume a.3-to 4-week shelf life for toluidine blue once in liquid state.

The purpose of the alcohol stage is not entirely clear. although it may serve to soften the MMA. In the original protocol, Schenk et al. (7) claim that both the formic acid 

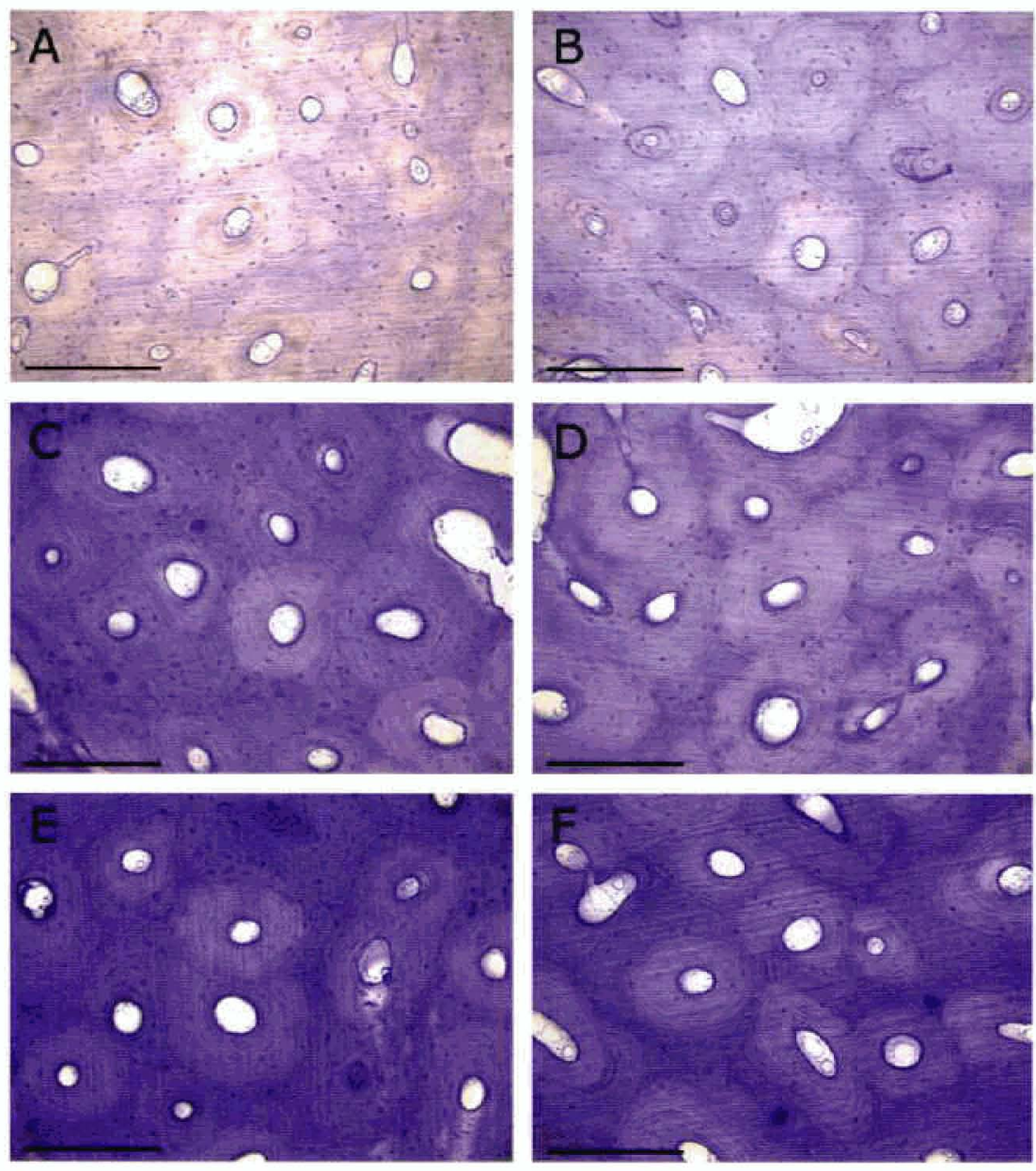

Figure 2. Photomicrographs of bone stained with toluidine blue taken with a $\times 10$ objective: scale is equivalent to 250 microns. A, femoral section stained while agitating during toluidine blue exposure only. B, femoral section stained while agitating at each step in the procedure, C, femoral section etched for 5 min and stained for 5 min with agitation ar all stages. D, femoral section etched for 5 min and stained for 15 min with agitation at all stages. E, femoral section etched for $10 \mathrm{~min}$ and stained for $5 \mathrm{~min}$ with agitation at all stages. F, femoral section etched for 10 min and stained for 15 min with agitation at all stages

and the alcohol act to etch the section. However, the original protocol used methanol, whereas in this investigation ethanol was used. Alcohol also softens the surrounding plastic, permitting the dye greater tissue access. Eurell et al. (15) suggest that etching sections embedded in methyl methacrylates with methanol resulted in cracking that impeded staining and analysis of the specimen. Alternatively, the alcohol stage may prevent cracking and partially dehydrate the osseous tissue that has been rinsed with distilled water, thereby increasing the absorption of toluidine blue by cement lines.

The findings of this study are consistent with Eurell et al., (15) who also found it necessary to etch undecalcified bone sections with acid before staining with toluidine blue $(\mathrm{pH}$ 7.0 to 8.0 ). Eurell et al. (15) did note that heating the toluidine blue solution allowed the researchers to omit the methanol etching that resulted in cracked sections. Interestingly, An and Martin (14) provide a protocol for the staining of bone sections using toluidine blue that uses a 3 -min hydration with distilled water, 30-min exposure to toluidine blue $(\mathrm{pH} 3.5), 5-\mathrm{min}$ rinse in distilled water, a dehydration series, and a clearing series. The An and Martin toluidine blue protocol is much simpler than what is proposed here, but it is unclear whether or not their protocol is for thin sections, thick sections, or both. The absence of an acid etching stage prior to staining in the An and Martin protocol is likely accounted for by the specific toluidine blue solution utilized in their protocol. The $\mathrm{pH}$ of the toluidine blue solution used by An and Martin was 3.5, where in the current analysis the solution was less acidic $(\mathrm{pH} 9.0)$. The $\mathrm{pH} 7.0$ to 8.0 toluidine blue solution used by Eurell et al. (15) more closely approximates the solution used in this analysis, which again speaks to the importance of etching of the calcified material prior to dye exposure.

It is possible that the fluids used to embalm the tissues in this analysis could have interacted with the solutions used in the protocol. Because the material used in this analysis was derived from several different medical science programs, it is not possible to determine what specific chemicals were used in the embalming process, or how much time passed between death and embalming. However, given that this procedure appears to adequately stain tissue derived from multiple sources, it is not likely that embalming fluids significantly influence staining success.

The shelf life of the toluidine blue solution appears to be approximately 3-4 weeks, at which point it loses its meta- 

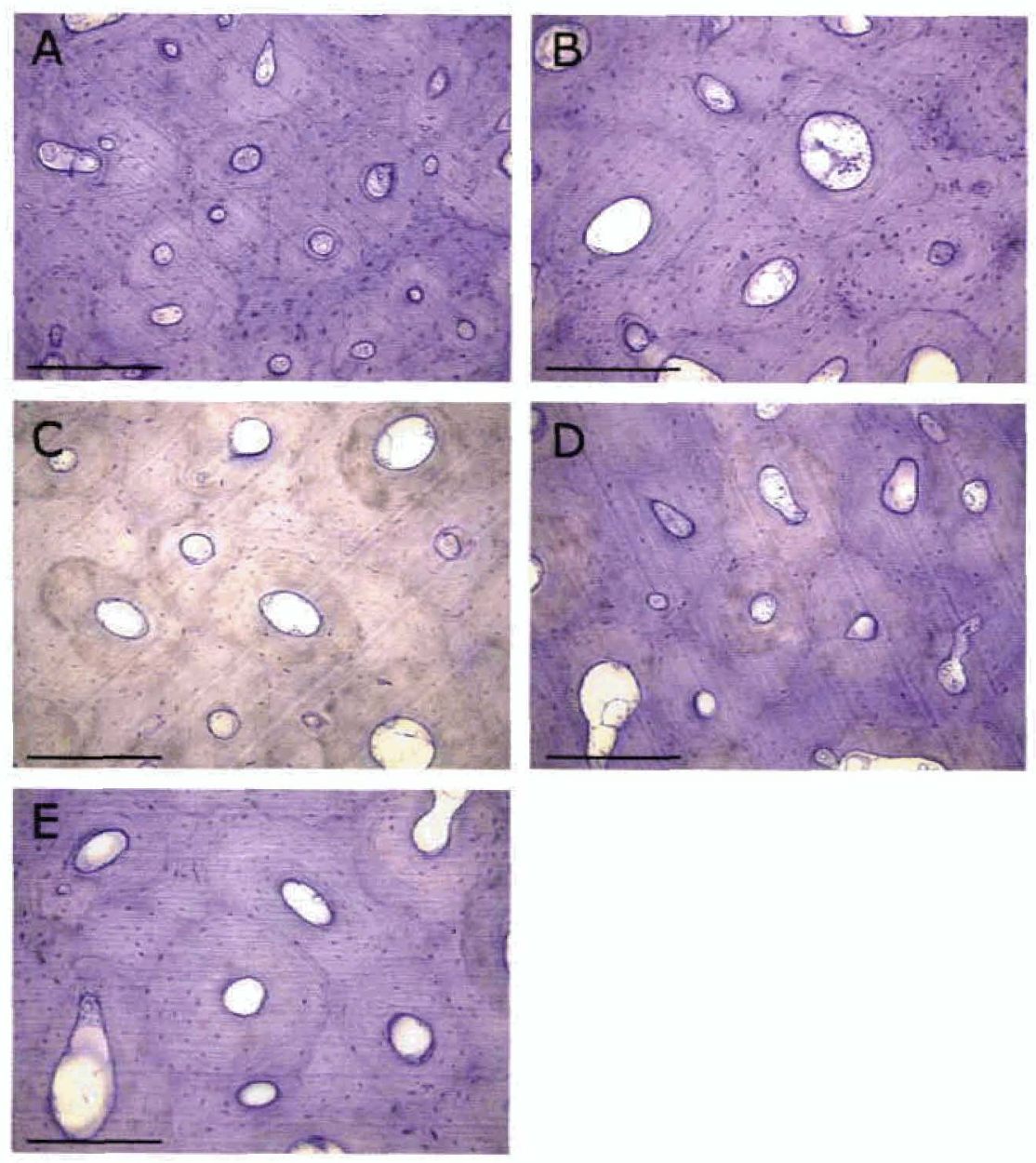

Figure 3. Photomicrographs of bone stained with toluidine blue taken with a $\times 10$ objective; scale is equivalent to 250 microns. A, femoral section etched for 3 min and stained for $5 \mathrm{~min}$ with agitation at all stages of the protocol. B, femoral section etched for $3 \mathrm{~min}$ and stained for $15 \mathrm{~min}$ with agitation at all stages of the protocol. C, femoral section etched for $2 \mathrm{~min}$ and stained for $5 \mathrm{~min}$ with agitation at all stages of the protocol. D. femoral section etched for $3 \mathrm{~min}$ and stained for $5 \mathrm{~min}$ with agitation at all stages of the protocol. E, femoral section etched for 4 min and stained for 5 min with agitation at all stages of the protocal.

Table 6. Protocol for the Staining of Cement Lines Using Toluidine Blue

\begin{tabular}{llc}
\hline Solution & $\begin{array}{c}\text { Immersion time } \\
\text { with agitation }\end{array}$ & $\begin{array}{c}\text { Immersion } \\
\text { time without } \\
\text { agitation }\end{array}$ \\
\hline (1) Formic acid $0.1 \%$ & $\begin{array}{c}3 \mathrm{~min} \\
\text { Quick rinse }\end{array}$ & $\begin{array}{c}5 \mathrm{~min} \\
\text { Quick rinse }\end{array}$ \\
(2) $\mathrm{d}_{2} \mathrm{O}$ & $15 \mathrm{~min}$ \\
(3) $70 \%$ EtOH & $15 \mathrm{~min}$ & $5 \mathrm{~min}$ \\
(4) Toluidine blue & & Quick dip \\
$1 \%$ solution & \\
(5) $\mathrm{dH}_{2} \mathrm{O}$ & $5 \mathrm{~min}$ & \\
(6) $70 \%$ EtOH & Quick dip & $30 \mathrm{~s}$ \\
$($ differentiation) & $30 \mathrm{~s}$ & $30 \mathrm{~s}$ \\
(7) $95 \%$ EtOH & $30 \mathrm{~s}$ & $30 \mathrm{~s}$ \\
(8) $100 \% \mathrm{EtOH}$ & $30 \mathrm{~s}$ & $30 \mathrm{~s}$ \\
(9) $100 \% \mathrm{EtOH}$ & $30 \mathrm{~s}$ &
\end{tabular}

"Toluidine blue solution at $1 \%$ toluidine blue, $1 \%$ sodium tetraborate hydrous.

chromatic properties. Some stains will expire simply because of overexposure to the air or light. Although the former could explain the shelf life of the solution used in this analysis, the latter likely does not as the solution was kept in a dark cabinet. A more likely explanation is that the solution became diluted with $70 \% \mathrm{EtOH}$ after serial usage. Unfortunately this was not controlled for, but likely explains why the solution used in this analysis only maintained its staining properties for 3-4 weeks.

Future research on toluidine blue will seek to compare results from both hydrated and anhydrous forms. Previous comparative tests between hydrated and anhydrous sodium borate shows anhydrous sodium borate gives slightly better definition of cement lines. In addition to comparing differences in the forms of toluidine blue, the influence of methanol on the staining success should also be considered. Furthermore, series analyses should be performed using the same protocol on sections of varying thickness from different bones, as variation in calcification will likely influence required etching time.

\section{Conclusions}

Etching of undecalcified bone sections is required for successful surface staining. Agitation during exposure to etching and staining solutions both increases staining intensity and uniformity. The suggested protocol(s) for staining cement lines in thick plastic sections, provided in Table 6 . suggests that use of agitation during the staining procedure 

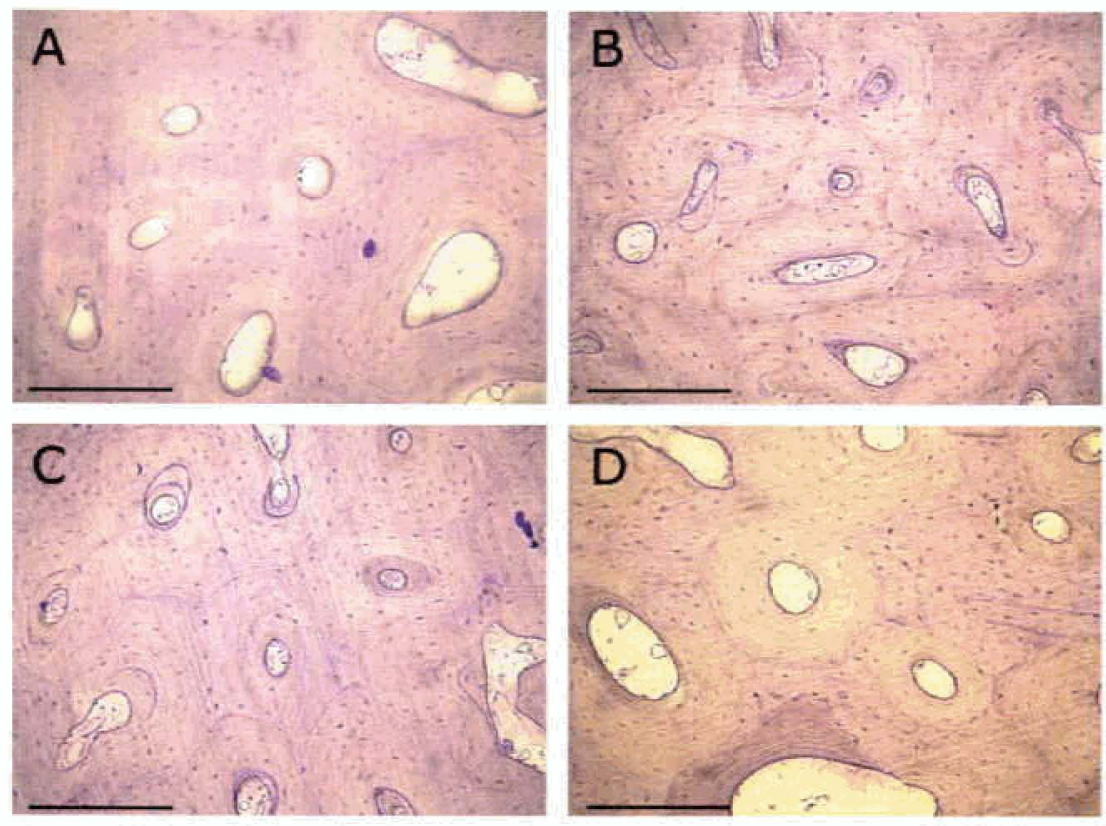

Figure 4. Photomicrographs of bone stained with toluidine blue taken with a $\times 10$ objective; scale is equivalent to 250 microns. A, femoral section not exposed to alcohol subsequent to staining. B, femoral section exposed to alcohol for 5 min subsequent to staining. C, femoral section exposed to alcohol for 10 min subsequent to staining. D, femoral section exposed to alcohol for 15 min subsequent to staining.

can reduce the required etching time by $2 \mathrm{~min}$. It is necessary, however, to examine the $\mathrm{pH}$ of the toluidine blue solution prior to staining, as increased acidity of this solution will likely require decreased acid or alcohol etching.

\section{Acknowledgments}

We thank David Burr for helpful comments on drafts of the manuscript. We also thank Keith Condon, who provided invaluable technical advice and assistance throughout this project. Finally, thanks to Taffy Hooser for technical assistance. This research was supported by the Department of Anatomy and Cell Biology, Indiana University School of Medicine, Indianapolis, IN.

\section{References}

1. Derkx P. Brikenhäger-Frenkel DH: A thionin stain for visualizing bone cells, mineralizing fronts and cement lines in undecalcified bone sections. Biotech Histochem 70:70-74. 1995

2. Villanueva AR, Hattner RS, Frost HM: A tetrachrome stain for fresh, mineralized bone sections, useful in the diagnosis of bone diseases. Stain Technol 39:87-94, 1964

3. Epker BN. Klein M, Frost HM: Magnitude and location of cortical bone loss in the human rib with aging. Clin Orthop 41:198-203. 1965

4. Frost HM: Staining of fresh undecalcified, thin bone sections. Stain Tech 34:135-146, 1959

5. Clarke DF: Histological and radiographic variation in the parietal bone in a cadaveric population. Master's thesis, Department of Anatomy, University of Queensland, 1987
6. Watanabe Y, Konishi M, Shimada M, Ohara H, Iwamoto S: Estimation of age from the femur of Japanese cadavers. Forensic Sci Int 98:55-65, 1998

7. Schenk RK. Olah AJ. Hermann W: Preparation of calcified tissues for light microscopy. In Methods of Calcified Tissue Preparation. Dickson GR (ed). Elsevier, New York. 1984. pp $1-56$

8. Burr DB: Estimated intracortical bone turnover in the femur of growing Macaques: implications for their use as models in skeletal pathology. Anat Rec 232:180-189, 1991

9. Havill LM: Osteon Remodeling Dynamics in the Rhesus Macaque: Normal Variation and the Genetic Contribution. PhD Dissertation, Department of Anthropology, Indiana University-Bloomington, 2002

10. Martin RB, Burr DB, Sharkey NA: Skeletal Tissue Mechanics. Springer, New York, 1998

11. Schaffler MB, Burr DB. Frederickson RG: Morphology of the osteonal cement line in human bone. Anat Rec 217:223-228. 1987

12. Burr DB, Martin RB. Schaffler MB, Radin EL: Bone remodeling in response to in vivo fatigue microdamage. $J$ Biomech $18: 189-200,1985$

13. Martin RB, Burr DB: A hypothetical mechanism for the stimulation of osteonal remodeling by fatigue damage. J Biomech 15:137-139, 1982

14. An YH, Martin KL: Handbook of Histology Methods for Bone and Carrilage. Humana Press, NJ. 2003

15. Eurell JAC. Sterchi DL: Microwaveable toluidine blue stain for surface staining of undecalcified bone sections. $J$ Histotechnol 17:357-359. 1994 